\title{
ECG Imaging of Simulated Atrial Fibrillation: Imposing Epi-Endocardial Similarity Facilitates the Reconstruction of Transmembrane Voltages
}

\author{
Steffen Schuler ${ }^{1}$, Danila Potyagaylo ${ }^{1}$, Olaf Dössel ${ }^{1}$ \\ ${ }^{1}$ Institute of Biomedical Engineering, Karlsruhe Institute of Technology (KIT), Karlsruhe, Germany
}

\begin{abstract}
Electrocardiographic imaging (ECGI) could help in diagnosis and treatment of atrial fibrillation $(A F)$, the most common life-threatening arrhythmia. Based on a previous work by Figuera et al. on the reconstruction of epicardial potentials (EP) during AF, we explore the performance of a Tikhonov regularization with two spatial constraints for transmembrane voltage (TMV) based ECGI. We develop a new method to impose epi-endocardial similarity and show its benefit for ECGI of atrial activity. Apart from $T M V s$, local activation times and dominant frequency are evaluated as target parameters. In the AF models studied, joint reconstruction of epi-and endocardial TMVs showed performance comparable to the previously reported EPS imaging.
\end{abstract}

\section{Introduction}

ECGI aims to non-invasively reconstruct the cardiac electrical activity from body surface potentials (BSP). Although ECGI is quite well validated for regular activities of the ventricles and some forms of ventricular arrhythmias, its application to irregular activity of the atria is fairly new. Recently, Figuera et al. [1] studied the performance of various reconstruction methods using computer models of AF. They reconstructed the potentials on the epicardial surface of the atria and found that among methods not requiring statistical a priori information, Tikhonov-based methods perform as well as more complex methods. In the present work, we use their computer models of atrial activity and perform reconstructions of TMVs using a Tikhonov regularization with two spatial constraints.

\section{Methods}

\subsection{Atrial activity and geometries}

We use the set of atrial excitation propagation simulations from [1], which consists of three cases:

1. Normal sinus rythm (SR): Periodical activation at the sinoatrial node with a cycle length of $0.8 \mathrm{~s}(1.25 \mathrm{~Hz})$.
2. Simple atrial fibrillation $(S A F)$ : A single functional reentry in the right atrium creates rotational activity at $7.3 \mathrm{~Hz}$. The left atrium is activated at $4.7 \mathrm{~Hz}$.

3. Complex atrial fibrillation $(C A F)$ : A single functional reentry near the right superior pulmonary vein (left atrium) creates rotational activity at $6.8 \mathrm{~Hz}$. The rest of the atria activates at $5.4 \mathrm{~Hz} .25 \%$ of cells are fibrotic.

We use 4 s (2000 timesteps) of all three simulations. From the surface of the tetrahedral mesh used for excitation simulations, a much coarser, regular triangle mesh with 4800 nodes is created for forward and inverse calculations, consisting of endo- and epicardial surfaces. Surface TMVs are transferred to the new mesh by averaging values within a radius of half the new edge length. The same triangle mesh as in [1] with 659 nodes (= electrodes) is used for the torso.

\subsection{Forward and inverse calculations}

Given equal anisotropy ratios of intra- and extracellular conductivities, the BSPs can be calculated from the transmembrane voltages on the atrial surface encompassing both the endo- and epicardial surfaces [2]. We use isotropic intra- and extracellular conductivies and an isotropic, homogenous torso model. The lead field matrix A is then obtained using the boundary element method, and BSPs $\mathbf{b}$ are calculated from TMVs $\mathbf{x}$ by

$$
\mathbf{b}=\mathbf{A x}+\varepsilon
$$

where $\varepsilon$ represents white gaussian noise. We tested three different signal-to-noise ratios (SNR): $\infty, 40$ and $20 \mathrm{~dB}$. As in [1], it is assumed that each electrode is affected by the same absolute noise power, which is set to the average signal power of all nodes divided by the SNR. BSPs for 40 and $20 \mathrm{~dB}$ are filtered before reconstruction using a zerophase, 4th-order low-pass Butterworth filter with a cutoff frequency of $30 \mathrm{~Hz}$. BSPs for SNR $=\infty$ are not filtered.

For solving the inverse problem, we use the same matrix A as for the forward calculation and thus neglect errors due to imperfect geometries and conductivities. Tikhonov regularization with zero- and second-order terms is applied as described in more detail in [3]: 


$$
\mathbf{x}=\arg \min _{\mathbf{x}}\left\{\|\mathbf{A x}-\mathbf{b}\|_{2}^{2}+\lambda^{2}\|\mathbf{L} \mathbf{x}\|_{2}^{2}+\eta^{2}\|\mathbf{x}\|_{2}^{2}\right\}
$$

$\mathbf{L}$ approximates the surface Laplacian. A "large" $\lambda$ constrains the solution to be smooth, while a "large" $\eta$ constrains its signal energy. The solution to (1) is:

$$
\mathbf{x}=\left(\mathbf{A}^{\top} \mathbf{A}+\lambda^{2} \mathbf{L}^{\top} \mathbf{L}+\eta^{2} \mathbf{I}^{\top} \mathbf{I}\right)^{-1} \mathbf{A}^{\top} \mathbf{b}
$$

The values for $\lambda$ and $\eta$ are determined using the L-surface [3]. We sample both directions of the parameter space in log scale using 10 samples / decade over at least 2 decades, thus computing at least 400 solutions of (2) per timestep. The L-surface (Fig. 1) is obtained by plotting the logarithm of the residual norm over the logarithms of the Laplacian and energy norms. The corner of the L-surface is then located as the point, where the gaussian curvature is maximal and the mean curvature is positive. Reconstructions are performed using the median ("global") as well as the individual $\lambda$ and $\eta$ determined for each timestep ("instantaneous"). For SNR $=\infty, \lambda$ and $\eta$ were both set to $10^{-10}$. This is the smallest value, for which (2) was solvable.

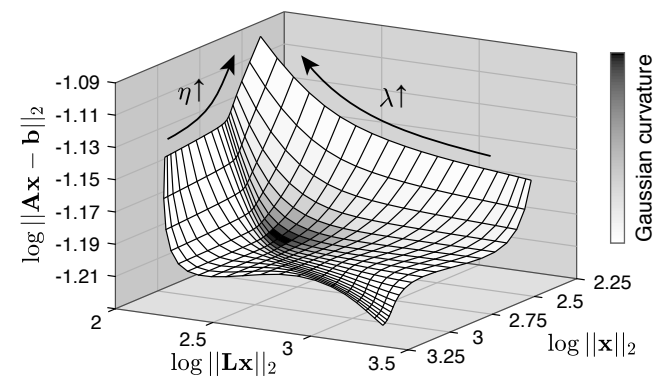

Figure 1. Typical L-surface (SAF, $20 \mathrm{~dB}, \mathrm{t}=2 \mathrm{~s})$.

\subsection{Epi-endo projection}

With a wall thickness of around $2 \mathrm{~mm}$, the atria are very thin. In the healthy case, the atrial walls therefore function much like a 2D surface for propagating excitation waves [4] and TMVs on the endocardial (endo) and epicardial (epi) side are usually very similar. In order to use this a priori information for inverse reconstructions, an "epi-endo projection" (EEP) is developed. The procedure is illustrated in Fig. 2. First, the surface mesh is divided into endo and epi surfaces containing 2099 and 2701 nodes, respectively. The outward-pointing normals are then calculated for each endo point and intersected with the epi surface to find the corresponding epi triangle. The value of the TMV at the intersection point is interpolated using barycentric coordinates and used as corresponding endo value. Having obtained the barycentric coordinates of all intersection points, the projection can be written in matrix form as:

$$
\mathbf{x}=\mathbf{P} \tilde{\mathbf{x}}
$$

$\mathbf{P}$ is an $M \times N$ matrix transforming $N$ epi values $\tilde{\mathbf{x}}$ to $M$ epi and endo values $\mathbf{x}$. If the first $N$ rows of $\mathbf{x}$ represent the epi values and the following $M-N$ rows represent the endo values, then the entries of $\mathbf{P}$ are given by

$$
p_{i j}= \begin{cases}1 & i=j \quad \text { with } i, j=1, \ldots, N \\ \alpha_{i j} & i=N+1, \ldots, M \text { and } j \in\left\{j_{1}, j_{2}, j_{3}\right\} \\ 0 & \text { otherwise }\end{cases}
$$

where $j_{1}, j_{2}, j_{3}$ denote the node indices of the epi triangle containing the projection of the endo node $i$ and $\alpha_{i j}$ is the barycentric coordinate of the projected endo node $i$ with respect to the epi node $j$.

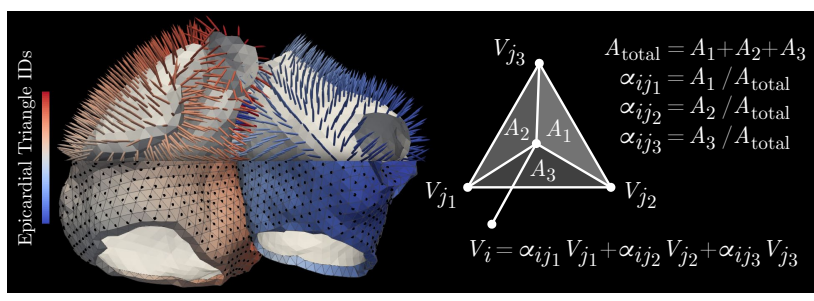

Figure 2. Epi-endo projection: Endo normals (top left). Epi intersection points (bottom left). The endo value $V_{i}$ is obtained from three epi values $V_{j}$ using interpolation.

The EEP can be used for reconstruction by obtaining a reduced lead field matrix for 'only epi nodes':

$$
\tilde{\mathbf{A}}=\mathbf{A P}
$$

After reconstruction, the values for both endo and epi nodes can be recovered with (3). In this work, we always use this direct application of the EEP.

As endo-epicardial dissociation can play a significant role in atrial fibrillation [4], a weaker form to prescribe similarity might be desired in future work. This can be achieved by using the EEP as additional regularization:

$$
\left\|\mathbf{P}_{\mathbf{D}} \mathbf{x}\right\|_{2}^{2} \quad \text { or }\left\|\mathbf{P}_{\mathbf{D}} \mathbf{x}\right\|_{1}
$$

$\mathbf{P}_{\mathrm{D}} \mathbf{x}$ are the differences of projected and non-projected endo TMVs. $\mathbf{P}_{\mathrm{D}}$ is obtained by horizontally concatenating the $M-N$ last rows of $\mathbf{P}$ with a negative identity matrix. $\mathbf{P}_{\mathrm{D}}$ can be extended to include both epi-to-endo and endoto-epi projections. Weighting its rows with the reciprocals of local epi-endo distances yields a method that has some similarity to the transmural regularization proposed in [5].

\subsection{Post-processing and metrics}

Similarity between true and reconstructed TMVs is measured using the Pearson correlation coefficient (CC) in two versions: The temporal CC is computed separately for each node using all time instants and the mean and standard deviation (SD) is computed across nodes, while the spatial CC treats nodes and time instants the opposite way.

Local activation times (LAT) for each node are extracted by detecting the peaks of the following signal [6]: 


$$
s(t)=\max \left\{\frac{g(t)}{\operatorname{std}(g(t))}, 0.5\right\}, \quad g(t)=\left\|\nabla V_{m}\right\|_{2} \frac{\partial V_{m}}{\partial t}
$$

For peak detection, a minimum peak prominence of 0.3 and a minimum peak distance of $0.1 \mathrm{~s}$ is prescribed (see MATLAB findpeaks). The same LAT detection is applied to true and reconstructed TMVs and similarity is assessed as follows. First, true positive activations are determined for each node by finding the nearest correspondences between the list of true LATs $T$ and the list of reconstructed LATs $R$. The items remaining in $T$ and $R$ after removing the true positives are defined as false negatives and false positives, respectively. Absolute errors (AE) between LATs of only true positive activations are calculated and the mean and SD of AEs is provided together with the false negative rate (FNR) and the false positive rate (FPR).

Dominant frequency $(D F)$ is determined as the reciprocal of the median interval of all detected LATs for a node. The median is chosen over the mean to reduce the sensitivity to false or missing activations. The relative absolute errors (RAE) between DFs determined from true and reconstructed TMVs are calculated and their mean and SD across nodes is given.

\section{Results}

\subsection{Effect of epi-endo projection}

Applying the EEP to reduce the lead field matrix reduces its condition number to one third. Errors made with the EEP itself were assessed by comparing the true endo values with the projected ones. From Table 1, it can be seen that the assumption of similar endo and epi TMVs holds very well for SR and SAF. For the CAF model containing fibrotic conditions, there are considerable errors. However, the assumption may still be deemed adequate, as inverse reconstruction errors are expected to be much larger.

Fig. 3 shows the effect of the EEP on reconstructions. TMVs were normalized for visualization by subtracting mean and dividing by SD (across all nodes and all timesteps). It can be seen that without EEP, endo TMVs are negatively correlated with true TMVs, while epi TMVs are positively correlated. This suggests that epi and endo TMVs partly compensate each other with respect to the electric field outside the atria, making it hard to find a unique solution to the inverse problem using independent endo and epi TMVs. Applying the EEP resolves this ambiguity and leads to higher CCs for both endo and epi TMVs.

Table 1. Deviation of projected from true endo TMVs. RE: Relative $L^{2}$-norm error. Temporal mean $\pm \mathrm{SD}$.

\begin{tabular}{|l|c|c|c|}
\cline { 2 - 4 } \multicolumn{1}{c|}{} & SR & SAF & CAF \\
\hline Spatial CC & $0.998 \pm 0.020$ & $0.996 \pm 0.002$ & $0.896 \pm 0.012$ \\
\hline Spatial RE $(\%)$ & $0.72 \pm 1.76$ & $3.76 \pm 0.88$ & $9.06 \pm 0.81$ \\
\hline
\end{tabular}

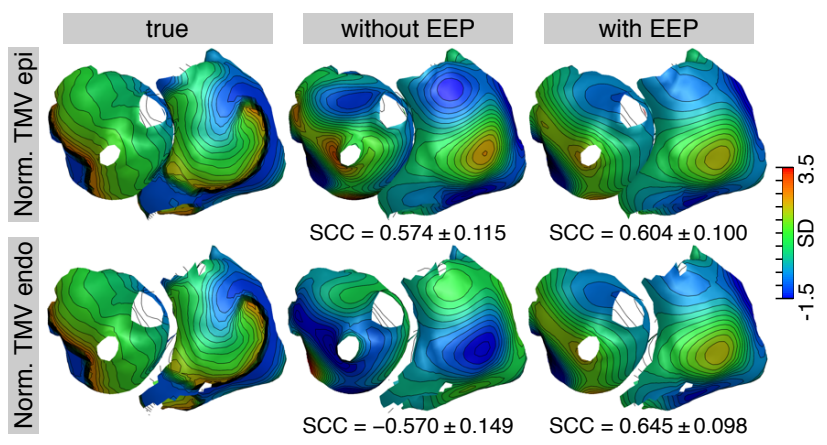

Figure 3. Reconstructions with and without EEP for SAF, $20 \mathrm{~dB}$, global $\lambda, \eta$. SCC: Spatial CC (mean \pm SD).

\subsection{Reconstruction results}

Fig. 4 depicts the time courses of regularization parameters for an SNR of $20 \mathrm{~dB}$. The repeated atrial activity can clearly be seen in these parameters. The more irregular the activation, the less important becomes the Laplacian term compared to the energy term and the smaller is the absolute change in both $\lambda$ and $\eta$, being largest for SR.

In the case of SR, instantaneous parameters perform better than the median as global parameter (Fig. 5). This is also reflected in the metrics (Fig. 6). For SR and both SNRs, TMV CCs (especially spatial CCs) are largest and LAT AEs are smallest with instantaneous parameters.

In the case of SAF and CAF, global parameters yield similar or slightly better results than instantaneous. Here, differences are largest for DF RAE in CAF. Reconstruc-

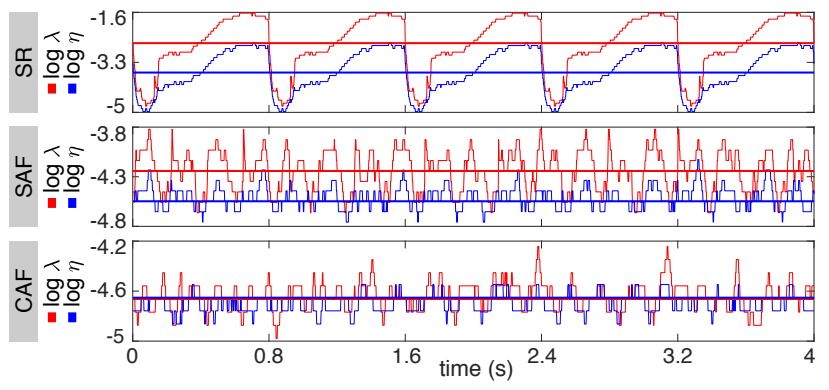

Figure 4. Regularization parameters determined using the L-surface for $20 \mathrm{~dB}$. Horizontal lines represent the median.

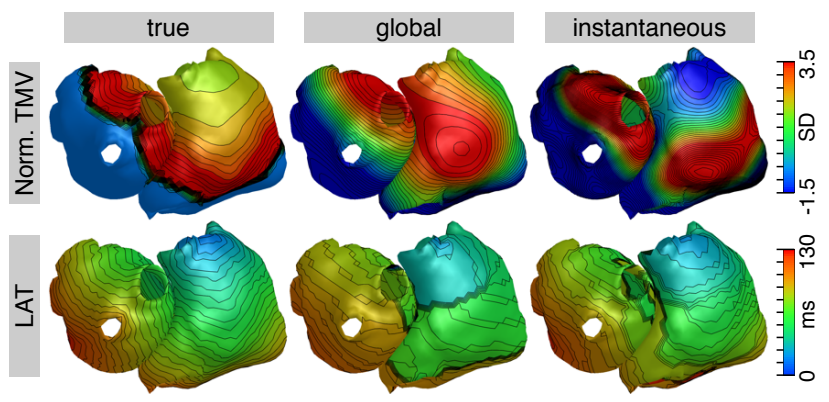

Figure 5. Global vs. instant. $\lambda, \eta$ for $\mathrm{SR}, 20 \mathrm{~dB}, \mathrm{t}=1.68 \mathrm{~s}$. 
tions for SAF and CAF are shown in Fig. 7 and 8. Without noise, TMVs, LATs and DFs are very similar to the ground truth. For $20 \mathrm{~dB}$, TMVs become more smoothed and LATs and DFs deteriorate especially in the interatrial region.
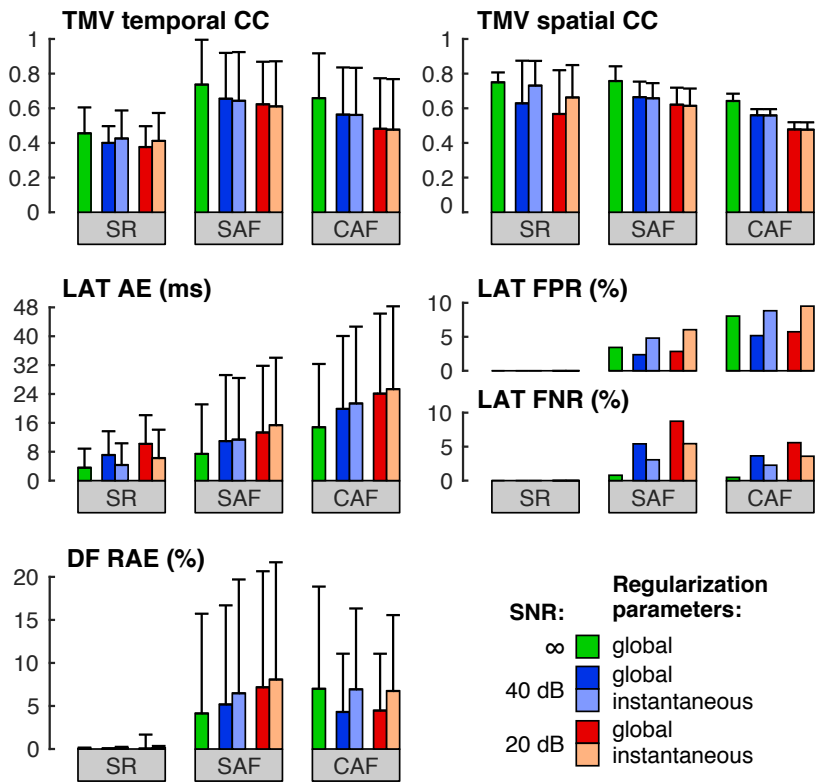

Figure 6. Performance metrics for TMVs, LATs and DF. Bars represent the mean and antennas the SD.

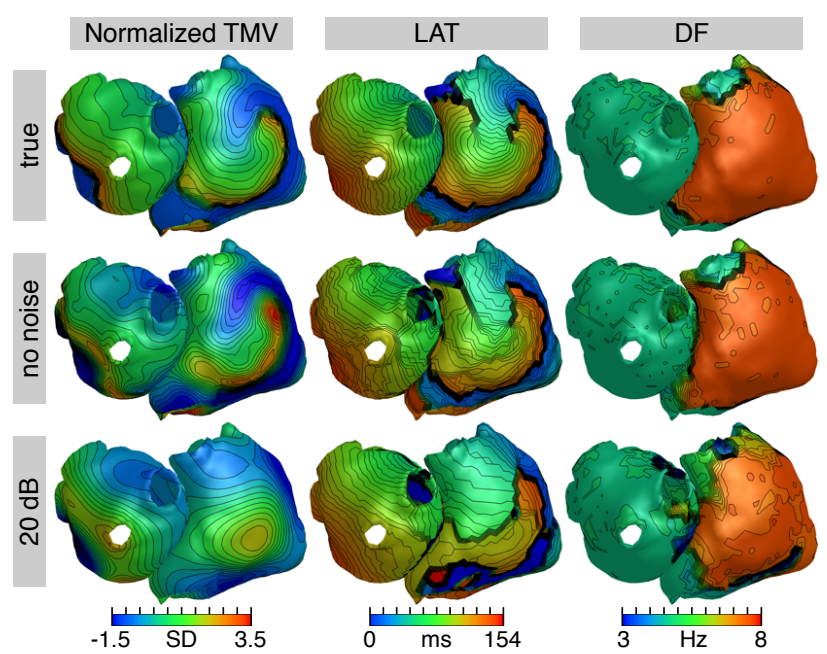

Figure 7. Reconstruction results for SAF with global $\lambda, \eta$.

\section{Discussion and conclusion}

TMV CCs for $20 \mathrm{~dB}$ in SAF and especially in CAF were slightly better than for EPs, while DF RAEs were slightly worse than reported in [1]. Overall performance of TMVbased reconstructions was therefore similar to Tikhonovbased reconstructions of EPs in [1]. In summary, we developed a new method to impose epi-endo similarity and successfully applied it to improve TMV-based ECGI of AF.

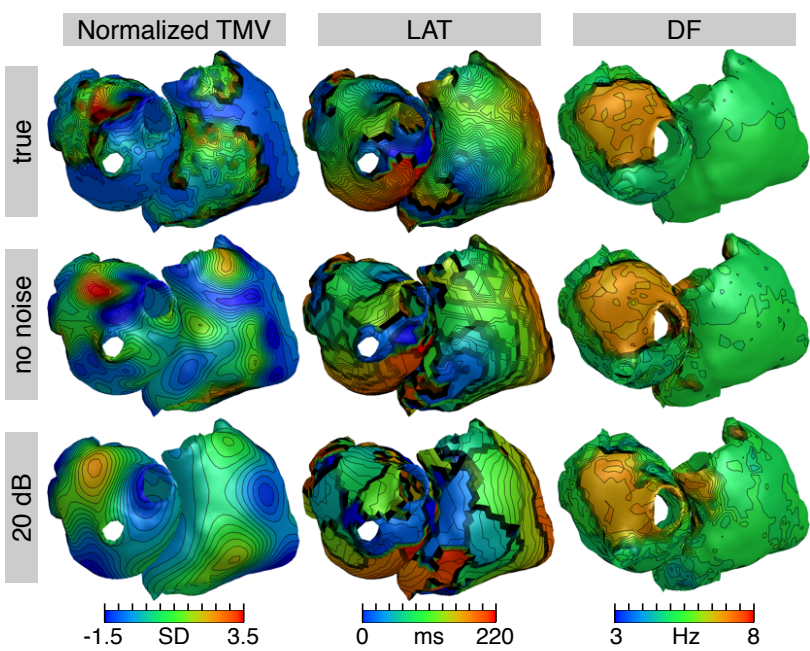

Figure 8. Reconstruction results for CAF with global $\lambda, \eta$.

\section{Acknowledgements}

We would like to thank María S. Guillem for kindly providing the simulations of atrial excitation propagation. For very helpful discussions and advice, we thank Lukas Baron, Gustavo Lenis and Nicolas Pilia.

\section{References}

[1] Figuera C, Suárez-Gutiérrez V, Hernández-Romero I, Rodrigo M, Liberos A, Atienza F, Guillem MS, Barquero-Pérez Ó, Climent AM, Alonso-Atienza F. Regularization techniques for ecg imaging during atrial fibrillation: A computational study. Frontiers in Physiology 2016;7:466.

[2] Geselowitz DB. Description of cardiac sources in anisotropic cardiac muscle: application of bidomain model. Journal of Electrocardiology 1992;25:65-67.

[3] Brooks DH, Ahmad GF, MacLeod RS, Maratos GM. Inverse electrocardiography by simultaneous imposition of multiple constraints. IEEE Trans Biomed Eng 1999;46(1):3-18.

[4] Verheule S, Eckstein J, Linz D, Maesen B, Bidar E, Gharaviri A, Schotten U. Role of endo-epicardial dissociation of electrical activity and transmural conduction in the development of persistent atrial fibrillation. Progress in Biophysics and Molecular Biology 2014;115(2):173-185.

[5] Erem B, Coll-Font J, Orellana RM, Štóvíček P, Brooks DH. Using transmural regularization and dynamic modeling for noninvasive cardiac potential imaging of endocardial pacing with imprecise thoracic geometry. IEEE Transactions on Medical Imaging 2014;33(3):726-738.

[6] Erem B, Brooks DH, van Dam PM, Stinstra JG, MacLeod RS. Spatiotemporal estimation of activation times of fractionated ecgs on complex heart surfaces. In Engineering in Medicine and Biology Society. IEEE, 2011; 5884-5887.

Address for correspondence:

Steffen Schuler, publications@kit.edu

Fritz-Haber-Weg 1, 76131 Karlsruhe, Germany 